\title{
Mind the gap! The Lucent Line Sign: A Pathognomonic Radiographic Finding in Periprosthetic Hip Fractures Around Cemented Polished Tapered Femoral Stems
}

\author{
Joanna L Maggs ${ }^{1}$, Eric W Swanton ${ }^{2}$, Matthew JW Hubble ${ }^{1}$, Matthew J Wilson ${ }^{1}$
}

\section{Learning Point of the Article:}

When considering the possible diagnosis of femoral periprosthetic fracture (PPF) in a patient with a total hip arthroplasty (THA) with a cemented polished tapered femoral stem, do not just rely on looking for a cortical breach on radiographs. The presence of a lucent line along the stem cement interface, the "Lucent Line Sign", is pathognomonic for a PPF and its recognition can avoid delays in diagnosis and treatment.

\section{Abstract}

Background: Diagnosing minimally displaced femoral PPFs using plain-film radiographs can be challenging. As a result, fractures can be missed.

Case Report: We present four cases of minimally displaced PPFs around cemented polished tapered stems. In each case, radiographs demonstrate no clear cortical breach, but the presence of a fracture is highlighted by a pathognomic lucent line between the cement mantle and the prosthesis: The Lucent Line Sign.

Conclusion: Paying attention to the Lucent Line Sign can be pivotal in the diagnosis of minimally displaced PPFs around cemented polished tapered stems.

Keywords: Periprosthetic fracture, hip fracture, femoral fracture, taper-slip stem, polished-tapered stem, radiographs.

\section{Introduction}

Periprosthetic femoral fractures (PPFs) after total hip arthroplasty (THA) are associated with significant morbidity. Managing them can be challenging and expensive $[1,2,3,4]$. As the number of primary THAs increases, so too does the burden of PPFs $[5,6]$.

PPFs are typically classified using the Vancouver classification. This groups them according to the site of the fracture, implant stability, and the quality of the bone stock [7]. Vancouver B fractures occur adjacent to, or just distal to, the stem. They are divided into $\mathrm{B} 1$ fractures where the stem remains well fixed to bone, B2 fractures where the stem is loose and B3 fractures with a loose stem and poor residual bone stock. While diagnosis is usually obvious, both clinically and radiographically, in cases with minimal displacement, fractures can be hard to identify.

We present four examples of minimally displaced Vancouver B PPFs around cemented polished tapered stems, where radiographs demonstrated no clear fracture line or cortical breach. In each case, the presence of a fracture was diagnosed due to a lucent line between the prosthesis and cement mantle. The "Lucent Line Sign" occurs because the prosthesis has rotated within the cement mantle of the fractured proximal femur, creating a gap at the stem-cement interface. For this separation to occur and the gap to appear, a fracture must have occurred. The presence of a lucent line is thus pathognomonic of a fracture. Paying attention to this sign will reduce the risk of misdiagnosing these important injuries.

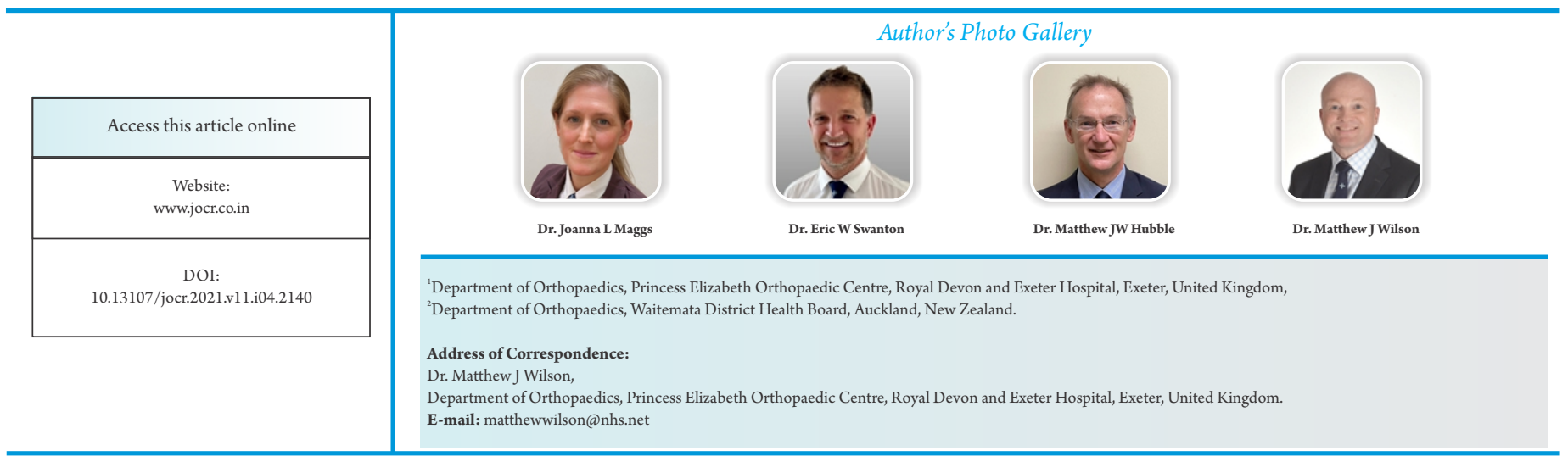

Journal of Orthopaedic Case Reports | pISSN 2250-0685 | eISSN 2321-3817 | Available on www.jocr.co.in | doi:10.13107/jocr.2021.v11.i04.2140 This is an Open Access article distributed under the terms of the Creative Commons Attribution Non-Commercial License (http://creativecommons.org/licenses/by-nc/3.0) which permits unrestricted non-commercial use, distribution, and reproduction in any medium, provided the original work is properly cited. 


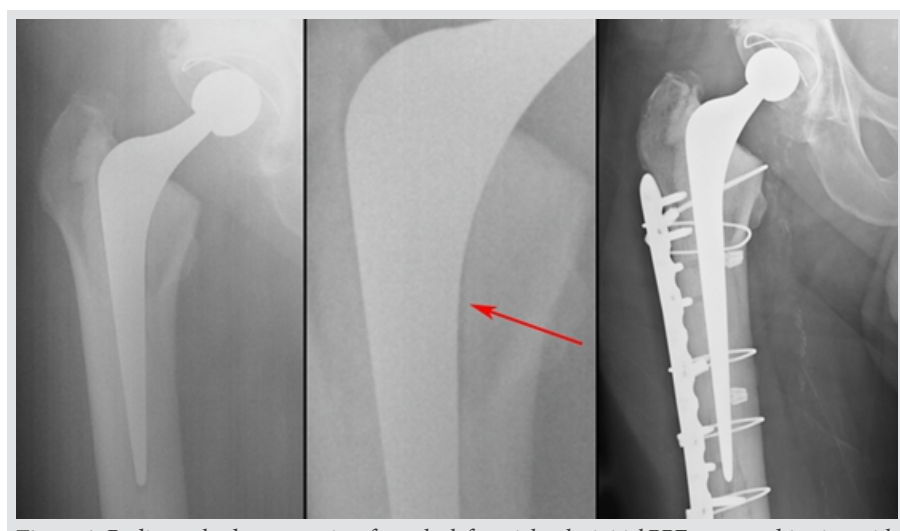

Figure 1: Radiographs demonstrating, from the left to right, the initial PPF, a zoomed-in view with an arrow to highlight the Lucent Line Sign, and the hip following revision surgery, of Case 1.

\section{Case Reports}

\section{Case 1}

This 80-year-old gentleman fell in the shower whilst visiting a relative in a different part of the country. He sustained a PPF around his 18-month-old THA and was transferred back to our unit in traction. At surgery, the stem was loose and had subsided, preventing reduction. The stem was removed, the fracture reduced and the well-fixed cement mantle bypassed distally to allow for cement-in-cement revision to a long Exeter stem. This was augmented with a 14-hole plate and cables. Two months post-operatively, he was walking with no aids, was pain free, and had a normal gait and a satisfactory range of movement. $\mathrm{He}$ continued to function well when last reviewed at 12 years postoperatively (Fig. 1).

\section{Case 2}

This gentleman had a left THA performed aged 46 for arthritis following slipped upper femoral epiphysis in childhood. Three years later, he fell from his bicycle landing heavily on his left side. He attended the Emergency Department where he was advised to mobilize with crutches and his radiographs were reported as showing no bony injury. A few days later, the patient telephoned the hip team. His X-rays were reviewed and the diagnosis of PPF was made due to the presence of the Lucent Line Sign. He

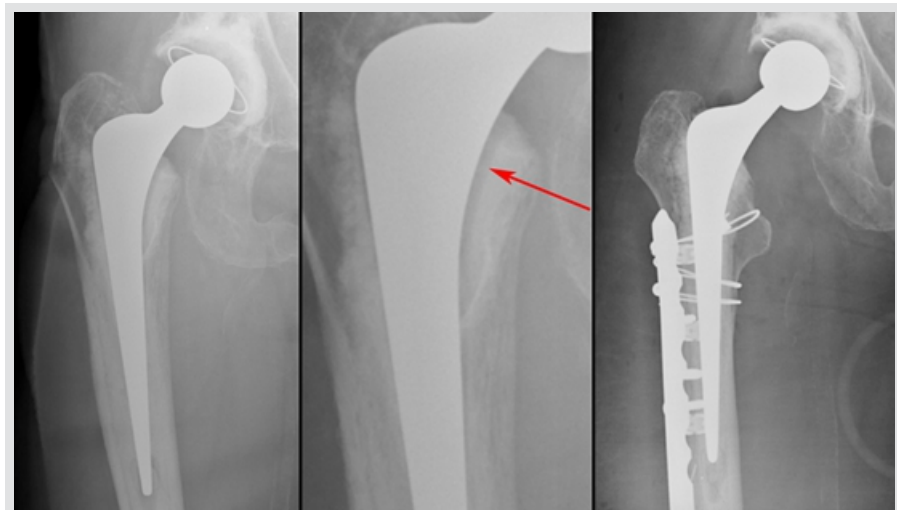

Figure 3: Radiographs demonstrating, from the left to right, the initial PPF, a zoomed-in view with an arrow to highlight the Lucent Line Sign, and the hip following revision surgery, of Case 3.

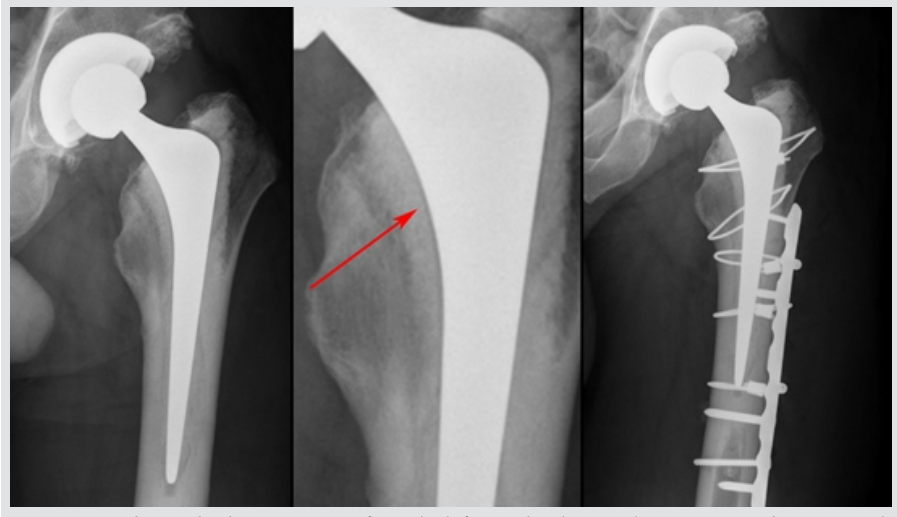

Figure 2: Radiographs demonstrating, from the left to right, the initial PPF, a zoomed-in view with an arrow to highlight the Lucent Line Sign, and the hip following revision surgery, of Case 2.

underwent open reduction and internal fixation of the fracture. At 6 months post-operatively, he felt he had regained full function and had returned to his active lifestyle. Three years later, he continues to manage 50-mile cycle rides and work as a rugby coach (Fig. 2).

\section{Case 3}

This 87-year-old woman sustained a fracture around a THA that had been previously been revised 10 years earlier for recurrent dislocation. At surgery, the fracture was irreducible due to the degree of subsidence and rotation. Therefore, the stem was removed, the fracture reduced with cables and a cement-in-cement revision undertaken with a standard Exeter stem supplemented with an anterolateral plate (Fig. 3).

\section{Case 4}

This 72-year-old gentleman fell 2 months following his primary THA. He attended the Emergency Department at the time, but the Lucent Line Sign was missed and he was discharged. He attended his routine post-operative review with the hip team, where the Lucent Line Sign was noted and a CT arranged, which delineated the fracture. Initially, he declined further surgical intervention, but was kept under close review. When his pain persisted, he opted to proceed with revision surgery 8

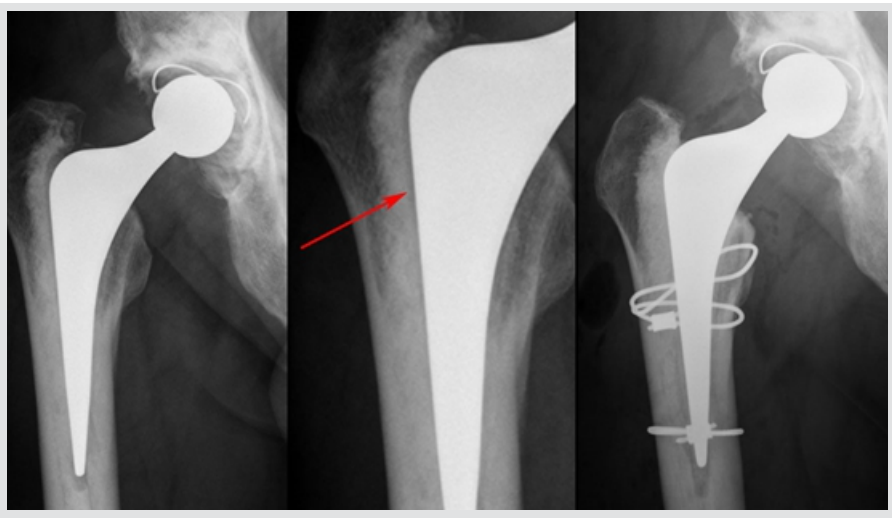

Figure 4: Radiographs demonstrating, from the left to right, the initial PPF, a zoomed-in view with an arrow to highlight the Lucent Line Sign, and the hip following revision surgery, of Case 4. 
months following his fall. At the time of operation, the stem was found to be loose, but the fracture was united and the majority of the cement bone interface remained well bonded. Two cables were positioned to support the healed fracture and a new stem was cemented into the original mantle, using the cement-incement technique (Fig.4).

\section{Discussion}

The mechanism of fracture around a cemented polished tapered stem has been likened to an axe or wedge splitting and fragmenting the proximal femur, with the twisting movement of the stem resulting in a spiral fracture $[8,9]$. Brew et al., in a biomechanical cadaveric study of the Exeter Femoral stem (Stryker, Mahwah), reproduced a Vancouver B fracture pattern using combined axial and rotational forces [10]. Rupprecht et al. studied the mechanisms of PPFs in cadaveric femurs with a cemented Exeter stem in situ [11]. They found that torsion led to pertrochanteric fractures, while lateral loading produced fractures closer to the tip of the stem, with the lateral loading mechanism requiring a lot more force to create a fracture than torsion.

The long spiral fractures that occur around cemented polished tapered stems, frequently pass posteriorly, making plain-film diagnosis challenging. While cross-sectional imaging and computer tomography can be useful, the suspicion of a fracture can often be confirmed by the presence of the Lucent Line Sign. As we have demonstrated, this radiolucent line is seen at the implant-cement interface on the lateral or, more typically,

\section{References}

1. Lindahl H. Epidemiology of periprosthetic femur fracture around a total hip arthroplasty. Injury 2007;38:651-4.

2. Lindahl H, Oden A, Garellick G, Malchau H. The excess mortality due to periprosthetic femur fracture. A study from the Swedish national hip arthroplasty register. Bone 2007;40:1294-8.

3. Drew JM, Griffin WL, Odum SM, van Doren B, Weston BT, Stryker LS. Survivorship after periprosthetic femur fracture: Factors affecting outcome. J Arthroplasty 2016;31:1283-8.

4. Bhattacharyya T, Chang D, Meigs JB, Estok DM 2nd, Malchau H. Mortality after periprosthetic fracture of the femur.J Bone Joint Surg Am 2007;89:2658-62.

5. Frenzel S, Vecsei V, Negrin L. Periprosthetic femoral fractures-incidence, classification problems and the proposal of a modified classification scheme. Int Orthop 2015;39:1909-20. medial border, and indicates a separation between the stem and the cement due to a rotation of the stem within the cement mantle. This separation is only possible in the presence of a PPF. It is important to note that a thin lucent line at the stem-cement interface over the shoulder of a polished tapered stem is a normal radiographic finding. It represents the normal taper-slip effect of subsidence that is a feature of this design of stem and is usually only one to two millimeters wide. It does not in itself represent a fracture but can be exaggerated in the setting of a fracture. In addition, it is worth noting that lucent lines associated with aseptic loosening of taper-slip stems occur at the cement-bone interface rather than cement-stem interface. Comparison with old radiographs may be helpful, but a high degree of clinical suspicion for fracture, in the presence of trauma, is paramount.

\section{Conclusion}

The cases presented in this report all demonstrate the presence of a lucent line along the stem cement interface. While it can be subtle, the Lucent Line Sign is pathognomonic for a PPF and its recognition can avoid delays in diagnosis and treatment.

\section{Clinical Message}

When considering the potential diagnosis of PPF in a patient with a THA with a cemented polished tapered femoral stem, do not just rely on looking for a cortical breach on radiographs. Look closely for the Lucent Line Sign.

6. Rocca D, Leung KS, Pape HC. Periprosthetic fractures: Epidemiology and future projections. J Orthop Trauma 2011;25 Suppl 2:S66-70.

7. Brady OH, Garbuz DS, Masri BA, Duncan CP. Classification of the hip. Orthop Clin North Am 1999;30:215-20.

8. Broden C, Mukka S, Muren O, Eisler T, Boden H, Stark A, et al. High risk of early periprosthetic fractures after primary hip arthroplasty in elderly patients using a cemented, tapered, polished stem. Acta Orthop 2015;86:169-74.

9. Grammatopoulos G, Pandit H, Kambouroglou G, Deakin M, Gundle R, McLardy-Smith P, et al. A unique periprosthetic fracture pattern in well fixed femoral stems with polished, tapered, collarless design of total hip replacement. Injury 2011;42:1271-6.

10. Brew CJ, Wilson LJ, Whitehouse SL, Hubble MJ, Crawford RW. Cement-in-cement revision for selected vancouver type B1 femoral periprosthetic fractures: A biomechanical 
analysis.J Arthroplasty 2013;28:521-5.

11. Rupprecht M, Sellenschloh K, Grossterlinden L, Püschel K,
Morlock M, Amling M, et al. Biomechanical evaluation for mechanisms of periprosthetic femoral fractures. J Trauma 2011;70:E62-6.
Conflict of Interest: Matthew Hubble and Matthew Wilson both

have contracts with Stryker

Source of Support: Nil

Consent: The authors confirm that informed consent was obtained from the patient for publication of this case report

\section{How to Cite this Article}

Maggs JL, Swanton EW, Hubble MJW, Wilson MJ.Mind the gap! The Lucent Line Sign: A Pathognomonic Radiographic Finding in Periprosthetic Hip Fractures Around Cemented Polished Tapered Femoral Stems. Journal of Orthopaedic Case Reports 2021 April;11(4):33-36. 